\title{
Sensitivity analysis of ST-segment epicardial potentials arising from changes in ischaemic region conductivities in early and late stage ischaemia
}

\author{
Barbara M Johnston $^{\mathrm{a}, *}$, Peter R Johnston ${ }^{\mathrm{a}}$ \\ ${ }^{a}$ School of Environment and Science, and Queensland Micro- and Nanotechnology Centre, Griffith University, Nathan, Queensland, \\ Australia, 4111
}

\begin{abstract}
Although computational studies are increasingly used to gain insight into diseases such as myocardial ischaemia, there is still considerable uncertainty about the values for many of the parameters in these studies. This is particularly true for the bidomain conductivity values that are used in normal tissue and, even more so, in ischaemic tissue, when modelling ischaemia. In this work, we extended a previous study that used a half-ellipsoidal model and a realistic model to study subendocardial ischaemia during the ST segment, so that we could simulate both early and late stage ischaemia. We found that, for both stages of ischaemia, there was still the same connection between the degree of ischaemia and the development of features such as minima and maxima in the epicardial potential distribution (EPD), although the magnitudes of the potentials were very often less, which may be significant in terms of detecting them experimentally.

Using uncertainty quantification associated with the ischaemic region conductivities, we also determined that the EPD features were sensitive to the ischaemic region extracellular normal and longitudinal conductivities during early stage ischaemia, whereas, during late stage ischaemia, the intracellular longitudinal conductivity was the most significant. However, since we again found that these effects were minor compared with the effects of fibre rotation angle and ischaemic depth, this might suggest that it is not necessary to use different conductivity values inside and outside the ischaemic region when modelling ST segment subendocardial ischaemia, unless the magnitudes of the potentials are an important part of the study.
\end{abstract}

Keywords: Early and late stage ischaemia, ST depression, Sensitivity analysis, Bidomain model, Gaussian Process emulators

\section{Introduction}

Detection of myocardial ischaemia via elevation and/or depression of the ST segment of the electrocardiogram (ECG) is one diagnostic method used by clinicians, despite averaged sensitivity and specificity values of around only $70-75 \%$ [1] for the presence of ischaemia.

Computer modelling studies have attempted to shed light on this by first considering the relationship between subendocardial ischaemia and depression and elevation of potentials on the epicardial surface. Connecting this work to the ECG would then require the addition of a torso model. These modelling studies have examined aspects such as the size, shape and location of the ischaemic region $[2,3,4,5]$, cardiac volume variability [6] and the effect of cardiac anisotropy $[7,8,2,9,10]$.

In recent years, the most commonly used model for cardiac electrical conduction is the bidomain model [11], although multidomain models have also been introduced [12]. In the bidomain model, the tissue is regarded as consisting of two interpenetrating domains, intracellular $(i)$

${ }^{*}$ Corresponding author and extracellular $(e)$, and the tissue properties are averaged over the two domains. Despite this approximation, the bidomain model is often successful in producing realistic electrograms and ECGs [13].

In the bidomain model, electrical conduction in the tissue occurs in three directions, two within the sheet of cardiac fibres, that is, longitudinally $(l)$ along the fibres and transversely $(t)$ at right angles to them, with the final direction normal $(n)$ to the sheet. Rotation of the sheets of fibres can also be taken in account via the conductivity tensor, which contains the six bidomain conductivity values $\left(g_{p q}, p=i, e, q=l, t, n\right)$.

Due to the experimental and computational difficulties associated with measuring and then extracting these conductivities, a consistent set of bidomain conductivities is still not available, even in normal tissue, and this leads to uncertainties in inputs and therefore outputs of computational models [14]. For example, various modelling studies $[15,8,2,9,10]$ have shown that changes in the conductivity values can lead to differences in the EPDs, when modelling subendocardial ischaemia under the assumption that the conductivity values are the same in both ischaemic and normal tissue.

However, based on previous studies, it seems likely 
that the conductivity values in ischaemic tissue will be different from those in normal tissue. This conclusion is based on the physical changes that occur during the time course of ischaemia (see the thorough summary in [16]). These changes can be categorised as occurring during three phases of ischaemia [16]: initial; early (approximately 5-20 minutes after the onset of ischaemia), and late (approximately 15-30 minutes after ischaemia onset).

Both the initial and early phases involve a drop in $g_{e l}$ $[17,16,7]$. Two mechanisms have been proposed for the initial drop: the collapse of the capillaries due to lack of blood flow [17], or, alternatively, the flow of fluid from the extracellular space into the capillaries due to a decrease in hydrostatic pressure [16]. The continued drop in $g_{e l}$ in the second (early) phase (plus a drop in $g_{e t}$ [7]) occurs because of a decrease in the volume of the extracellular space due to fluid flowing from the extracellular space into the myocytes [18]. In the third (late) phase, gap junctions close leading to a sharp decrease in intracellular conductivity [18].

These reductions in conductivity values in ischaemic tissue for the early and late stages of ischaemia are not easy to translate to reductions in different directions in the intracellular and extracellular domains and hence various studies have quantified them differently. For example, the values used by Boccia et al [19], which are based on the work of Kleber et al. [17], involve a $40 \%$ reduction in $g_{e l}$ and $g_{e t}$ and a $50 \%$ reduction in $g_{i l}$ and $g_{i t}$ at 20 minutes (i.e. late stage ischaemia). Also for late stage ischaemia, Barnes [20] applied a $40 \%$ reduction in $g_{e l}$ with a $90 \%$ reduction in $g_{i l}$ and no other changes. In addition, Hopenfeld et al. [7] modelled both early and late stage ischaemia using the following (normalised) longitudinal and transverse reductions: early - $g_{e l}$ (reduced from 1 to $1 / 2$ ) and $g_{e t}$ (from $1 / 3$ to $1 / 4$ ), and late - as for early for $g_{e l}$ and $g_{e t}$, with $g_{i l}$ (from 1 to $1 / 10$ ) and $g_{i t}$ (from $1 / 20$ to $1 / 1000)$. To the best of the authors' knowledge no information is given in any studies about the effect of ischaemia on the normal direction conductivities.

Given the lack of knowledge about the bidomain conductivity values in both normal and ischaemic tissue, and particularly in the normal direction, an uncertainty quantification (UQ) approach is ideal for determining the sensitivity of the EPDs to the model inputs. In that approach, an emulator (a fast-running statistical model of the original model) is constructed. This has the advantage of a large reduction in the number of model runs, with different sets of inputs, that would be required with a MonteCarlo approach. Examples of UQ methods are Gaussian Process emulators [21, 22], partial least squares [23, 24] and generalised polynomial chaos [25, 26].

This work is an extension of our previous work [10], which examined EPDs to determine the most significant inputs to a half-ellipsoidal model of acute ST segment subendocardial ventricular ischaemia, under the assumption that the conductivities in the ischaemic region were the same as in the normal tissue. The improvement in the simulations conducted here is that the bidomain conduc- tivities in the ischaemic region can be varied independently from the conductivities in the rest of the tissue. We apply this approach to model not just early (acute) stage ischaemia, but also late stage ischaemia, to see if the previously found qualitative progression $[3,10]$ of the EPD with increasing ischaemic depth (single minimum $\rightarrow$ maximum plus minimum $\rightarrow$ maximum flanked by two minima) still holds in these two scenarios. We also systematically investigate the effect that uncertainty in the values for the ischaemic region conductivities has on the EPDs that are produced, including finding the inputs to which the features of the EPD (e.g. minima and maxima) are the most sensitive.

\section{Methods}

\subsection{Modelling}

In this work, we simulate the effect of ST segment ischaemia in both a half-ellipsoidal model (Figure 1) and a more realistic model (Figure 2) of the left ventricle. The half-ellipsoid comes from the equations

$$
x=a \cos \theta \cos \phi, \quad y=a \cos \theta \sin \phi, \quad z=c \sin \theta
$$

with $0 \leq \theta \leq \pi / 2$ and $-\pi \leq \phi \leq \pi, a=2 \mathrm{~cm}, c=4 \mathrm{~cm}$ for the endocardial surface and $a=3 \mathrm{~cm}, c=5 \mathrm{~cm}$ for the epicardial surface (Figure 1). In this model, the cardiac fibres, which are offset by $-45^{\circ}$ on the epicardium [27], rotate linearly through a fibre rotation angle (ROT), given in degrees, between the epicardium and the endocardium $[28]$.

The 'realistic model' (Figure 2) uses a whole heart canine geometry [29], including a septum, and blood-filled ventricles that are open to the air at the base (as is the case for the half-ellipsoidal model). In both models, the ischaemic region is represented by a 'rectangular' shaped patch (see Figures 1 and 2) of ischaemic depth (ISC) (expressed as a $\%$ of the ventricular wall that extends outwards from the endocardium). More detail is given about the models in [10].

In both models, the electric potential, during the ST segment of the ECG, is simulated in the cardiac tissue via the passive bidomain equation $[11,27]$

$$
\nabla \cdot\left(\mathbf{M}_{i}+\mathbf{M}_{e}\right) \nabla \phi_{e}=-\nabla \cdot \mathbf{M}_{i} \nabla \phi_{m}
$$

while the potential in the blood obeys Laplace's equation $\nabla^{2} \phi_{b}=0$. Here $\phi_{m}=\phi_{e}-\phi_{i}$ and $\phi_{h}(h=i, e, m, b)$ represents the intracellular, extracellular, transmembrane and blood potentials, respectively, and $\mathbf{M}_{p}=\mathbf{A G}_{p} \mathbf{A}^{T}(p=$ $i, e)$ are conductivity tensors that combine the bidomain conductivities $\left(g_{p q}, p=i, e, q=l, t, n\right)$ with a rotation matrix $\mathbf{A}$, which maps the local fibre direction into the global coordinate system [27]. 


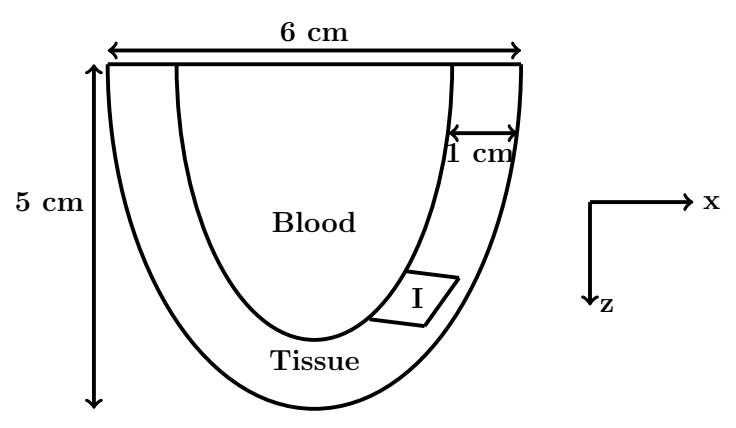

Figure 1: Half-ellipsoidal model - cross-sectional view $(x-z$ plane at $y=0$ ) with ischaemic region marked as $\mathrm{I}$.
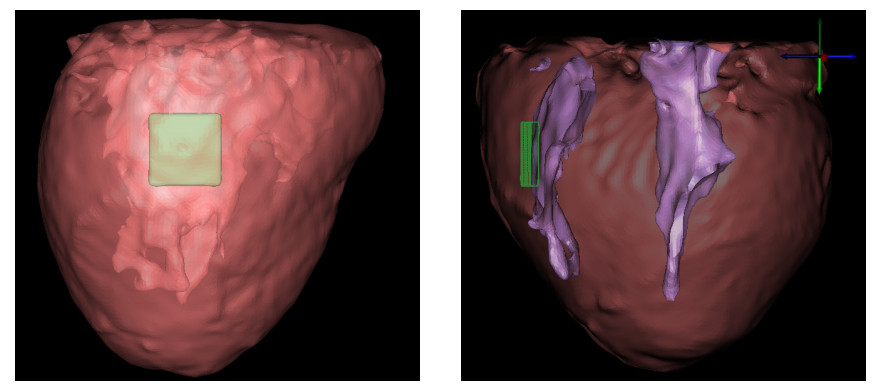

Figure 2: The realistic heart with the (a) ischaemic region (front view) and (b) ischaemic region (side view) and ventricles.

In this work, we consider two different representations for the transmembrane potential, which during the ST segment, we represent by [11]

$$
\phi_{m}(r, \theta, \phi)=\Delta \phi \Psi_{c}\left(r_{a}-r\right) \Psi_{c}\left(\theta-\theta_{0}\right) \Psi_{c}(\phi)
$$

where $\Delta \phi=-30 \mathrm{mV}$ is the difference between the plateau potentials in normal and ischaemic tissue. Here the ischaemic region is centred at $\left(r_{a}, \theta_{0}, 0\right)$ on the endocardial surface, and $\left(\Psi_{c}, c=1,2\right)$ are the two representations for the transmembrane potential that are given below. The version used previously $[30,27,20,10]$ is

$$
\Psi_{1}(t)= \begin{cases}\frac{1-\exp \left(-a_{t} / \lambda_{t}\right) \cosh \left(t / \lambda_{t}\right)}{1-\exp \left(-a_{t} / \lambda_{t}\right)} & |t| \leq a_{t} \\ \frac{\exp \left(-|t| / \lambda_{t}\right) \sinh \left(a_{t} / \lambda_{t}\right)}{1-\exp \left(-a_{t} / \lambda_{t}\right)} & |t|>a_{t}\end{cases}
$$

where $a_{t}(t=r, \theta, \phi)$ is the half-width of the ischaemic region and we set $\lambda_{t}=0.01$ for $t=r, \theta, \phi$, which produces $[27,10]$ a narrow border zone (Figure 3 ).

In order to more closely match experimental results [31, $2,32]$, we also use a 'new' version of the transmembrane potential $\Psi_{c}(t)[10]$, given by

$$
\Psi_{2}(t)= \begin{cases}\frac{1-2\left(1-a_{2}\right) \exp \left(-a_{1} / b_{1}\right) \cosh \left(t / b_{1}\right)}{1-2\left(1-a_{2}\right) \exp \left(-a_{1} / b_{1}\right)} & |t| \leq a_{1} \\ \frac{2 a_{2} \exp \left(-|t| / b_{2}\right) \sinh \left(a_{1} / b_{2}\right)}{1-2\left(1-a_{2}\right) \exp \left(-a_{1} / b_{2}\right)} & |t|>a_{1}\end{cases}
$$

where $a_{1}=0.9, b_{1}=0.2, b_{2}=0.01, a_{2}=0.1$ and $\Psi_{2}$ replaces $\Psi_{1}$ in equation (5) in the $t=\theta$ and $\phi$ directions (see Figure 3).

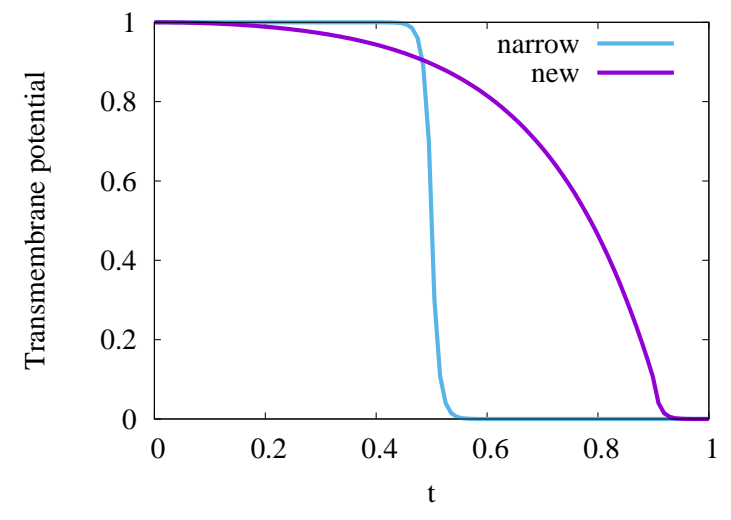

Figure 3: Transmembrane potential for the 'narrow' border zone and its 'new' representation (equation (5)).

To solve the model, we apply the same boundary conditions as previously $[27,10]$; that is, it is assumed that there is no current flux at the boundaries of the intra- and extracellular domains, nor at the blood-air and tissue-air interfaces, and that the extracellular current and potential are continuous at the blood-tissue boundary. We solve both models with previously validated techniques [33, 27, 20] that are based on the finite volume method. See [10] for mesh and element details.

\subsection{Simulating early and late stage ischaemia}

In this work, we examine the effect of using different (lower) values for the bidomain conductivities in the ischaemic region, compared with the rest of the tissue. We consider early and late stage ischaemia separately, and we model these scenarios by reducing, in the ischaemic region only, the extracellular conductivities (for early stage ischaemia), and then all six bidomain conductivities (for late stage ischaemia).

We initially apply the same reductions as in work by Hopenfeld et al. [7], and, in lieu of any information about conductivity changes in the normal direction, we apply the same changes in the normal direction as in the transverse direction. However, in a later study we also allow the reductions to vary independently over a wide range to examine which ischaemic region conductivities have the most significant effect on the EPDs. These fractional reductions in the ischaemic region are denoted $\left(f_{p q}, p=i, e, q=\right.$ $l, t, n)$, where each of these corresponds to a reduction of its corresponding conductivity $\left(g_{p q}\right)$ outside the ischaemic region. For example, if $g_{e l}=2.4 \mathrm{mS} / \mathrm{cm}$ and $f_{e l}=0.5$, then the extracellular longitudinal conductivity inside the ischaemic region is $1.2 \mathrm{mS} / \mathrm{cm}$. The values for the fractional reductions for early and late stage ischaemia [7] are given in the top row of Table 1.

\subsection{Analysing EPDs}

Once we have a set of inputs (for example, the mean values [10] in Table 2), to either the half-ellipsoidal or re- 
Table 1: Parameter values, or ranges of values, used in the Early and Late stage ischaemia simulations in the given Sections. The parameters $f_{p q}$ represent fractional reductions (in the ischaemic region) in the corresponding bidomain conductivities $g_{p q},(p=i, e, q=l, t, n), \mathrm{ROT}$ is fibre rotation angle and ISC is ischaemic depth. Where parameters are not mentioned they are set to their mean value as given in Table 2.

\begin{tabular}{l|l|l}
\hline Sections & Stage & Parameter values or ranges \\
\hline $3.1,3.2$ & Early & $f_{\text {el }}=0.5, f_{\text {et }}=f_{\text {en }}=0.25$ \\
& Late & $f_{i l}=0.9, f_{e l}=0.5, f_{i t}=f_{\text {in }}=0.98, f_{\text {et }}=f_{\text {en }}=0.25$ \\
\hline $3.3 .1,3.3 .2$ & Early & $f_{e q} \in[0.1,0.9], \mathrm{ISC}=10 \%, 20 \%, 60 \%$ \\
& Late & $f_{p q} \in[0.1,0.9], \mathrm{ISC}=10 \%, 20 \%, 60 \%$ \\
\hline 3.4 & Early & $f_{e q} \in[0.1,0.9], \mathrm{ROT} \in\left[60^{\circ}, 140^{\circ}\right], \mathrm{ISC} \in\left[10^{\circ}, 40^{\circ}\right], \mathrm{ISC} \in\left[40^{\circ}, 90^{\circ}\right]$ \\
& Late & $f_{p q} \in[0.1,0.9], \mathrm{ROT} \in\left[60^{\circ}, 140^{\circ}\right], \mathrm{ISC} \in\left[10^{\circ}, 40^{\circ}\right], \mathrm{ISC} \in\left[40^{\circ}, 90^{\circ}\right]$ \\
\hline 3.5 & Early & $f_{e l}=0.5, f_{e t}=f_{e n}=0.25, g_{p q} \in(\operatorname{mean} \pm 50 \%)$, \\
& ROT $\in\left[60^{\circ}, 140^{\circ}\right], \mathrm{ISC} \in\left[10^{\circ}, 40^{\circ}\right], \mathrm{ISC} \in\left[40^{\circ}, 90^{\circ}\right]$ \\
& Late & $f_{i l}=0.9, f_{e l}=0.5, f_{i t}=f_{i n}=0.98, f_{e t}=f_{e n}=0.25, g_{p q} \in(\operatorname{mean} \pm 50 \%)$, \\
& $\operatorname{ROT} \in\left[60^{\circ}, 140^{\circ}\right], \mathrm{ISC} \in\left[10^{\circ}, 40^{\circ}\right], \mathrm{ISC} \in\left[40^{\circ}, 90^{\circ}\right]$ \\
\hline
\end{tabular}

alistic model, we solve the model using the solution technique of Section 2.1 to produce EPDs. Examples of EPDs for the half-ellipsoidal model are given in Figure 4, where the EPDs are presented as polar plots. In these plots, the ellipsoidal surface is flattened to make a circle, where the apex is placed at the centre. This is achieved by mapping each point $(r, \theta, \phi)$ on the ellipsoidal surface to a point on the circle, where we ignore the $r$ component and give each node on the circle a radius that is determined by its value of $\theta$ and an angle that is determined by its value of $\phi$ in equation (1). In addition, we set $\phi_{e}$ to zero at the apex of the ventricle.

The EPDs, for both the half-ellipsoidal or realistic model, have features such as maxima or minima and it is these features that are the outputs of interest in this work. For example, consider Figure 4, which demonstrates that conductivity changes in the ischaemic region can change the character of the EPD. The EPD is produced for $\mathrm{ISC}=20 \%$ using the mean values in Table 2 throughout the tissue (a) or reducing $g_{i l}$ in the ischaemic region by $60 \%$ (i.e. $\left.f_{i l}=0.6\right)(\mathrm{b})$. In (a) there is a maximum over the ischaemic region, flanked by a minimum; however, in (b), reducing the intracellular longitudinal conductivity in the ischaemic region changes the EPD so that only the minimum remains over the ischaemic region.

In addition to examining sets of EPDs that are produced using particular conductivity values to simulate early and late stage ischaemia (row 1 of Table 1), we also systematically vary the values for the conductivities in the ischaemic region, and examine their effect on the various features of the half-ellipsoidal model (rows 2 and 3 of Table $1)$.

We achieve this by using the Latin Hypercube sampling routine in the package GP_emu_UQSA (DOI 10.5281/zenodo.215521) to produce sets of three $\left(f_{\text {eq }}, q=1, t, n\right)$ values for simulating early stage ischaemia and sets of six $\left(f_{p q}, p=i, e, q=1, t, n\right)$ for late stage ischaemia. We combine these with mean values from Table 2 for the other parameters and solve the model to produce design data;
Table 2: Mean values for parameters in this study. Units are degrees for fibre rotation (ROT), \% depth for ischaemic depth (ISC) and $\mathrm{mS} / \mathrm{cm}$ for conductivities.

\begin{tabular}{ccccccccc}
\hline ROT & ISC & $g_{b}$ & $g_{i l}$ & $g_{e l}$ & $g_{i t}$ & $g_{e t}$ & $g_{\text {in }}$ & $g_{\text {en }}$ \\
\hline 100 & 50 & 6.5 & 2.4 & 2.4 & 0.24 & 1.6 & 0.1 & 1.0
\end{tabular}

that is, sets of values for outputs such as the potential of the minimum in Figure 4.

We then analyse the sensitivity of these outputs to the inputs, using both partial least squares regression (PLS) and by constructing Gaussian Process (GP) emulators. Both these techniques are described in detail in previous papers $[10,9,34]$. Briefly, PLS $[23,24]$ finds a set of components that maximises the covariance between the outputs and inputs, while simultaneously decomposing both the output and input vectors. In the GP approach [21, 34], we fit an emulator to the design data using a Gaussian covariance function and a linear mean, using the software GP_emu_UQSA (DOI 10.5281/zenodo.215521). From this, we calculate main effect sensitivity indices to quantify the contribution of the inputs $\mathbf{x}$ to a particular output $y=$ $f(\mathbf{x})$.

Sensitivity, which is unsigned, is defined as

$$
\text { Sensitivity }=\frac{\operatorname{Var}\left[E\left\{f(x) \mid x_{w}\right\}\right]}{\operatorname{Var}\{f(x)\}} .
$$

where Var=variance, $E=$ expectation and $x_{w} \in \mathbf{x}$.

\section{Results}

\subsection{Mean EPDs for early and late stage ischaemia for the half-ellipsoidal model}

In previous work [10], initially we considered mean EPDs for the half-ellipsoidal model (that is, those where the parameters are set to the mean values in Table 2) and the way in which these varied with ischaemic depth. In 


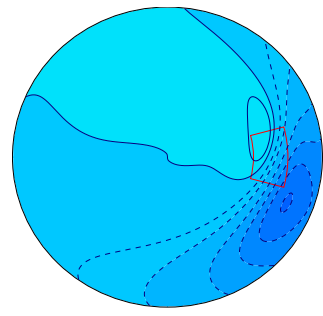

(a) Mean

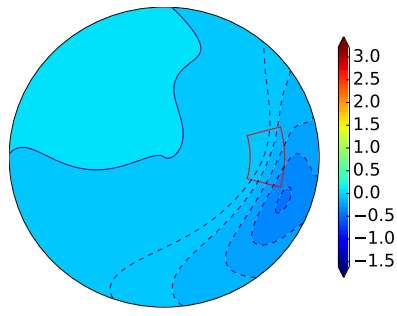

(b) $f_{i l}=0.6$
Figure 4: Reducing $g_{i l}$ in the ischaemic region by $60 \%$ (i.e. $f_{i l}=$ $0.6)$ for ischaemic depth $=20 \%$ changes the character of the EPD. The ischaemic region is outlined in red. Dashed lines are negative potentials and solid lines zero and positive potentials. Scale is in $\mathrm{mV}$. Contours are at $-0.9,-0.8, \ldots,-0.1,-0.05,0,0.025,0.05,0.25$, $0.5, \ldots, 1.25 \mathrm{mV}$

that work, the conductivities were taken to be the same both inside and outside the ischaemic region. In the current work, those results will be contrasted with studies where the conductivities are different within the ischaemic region.

For the mean EPDs (Figure 5, top two rows labelled Mean), we found that we could identify three types of EPDs: type 1 - a single minimum (min1) over the ischaemic region (Figure 5(a)); type 2 - a maximum (max) over the ischaemic region plus a single minimum (min1) (Figure 5(b)), and type 3 - a maximum (max) over the ischaemic region flanked by two minima (min1 and min2) (Figure 5(c)-(h)). We introduced the notation min1V, $\max \mathrm{V}$ and $\min 2 \mathrm{~V}$, for the potentials corresponding to min1, $\max$ and $\min 2$, respectively, and also angmin1, angmax and angmin2, respectively, for the angles made by these features, as shown in Figure 6.

The potentials $\min 1 \mathrm{~V}, \max \mathrm{V}$ and $\min 2 \mathrm{~V}$, associated with the mean plots in Figure 5 are given in the top of Table 3 (left column), where it can be seen that the magnitude of each of these potentials increases with increasing ischaemic depth (ISC).

We now consider again using mean values (Table 2) for the various parameters, but we adjust the conductivities in the ischaemic region by reducing them via the $f_{p q}$ values given in the top row of Table 1, so as to model either early or late stage ischaemia. Then we produce sets of EPDs similar to those for the mean case above. The set of EPDs corresponding to early stage ischaemia is given in rows three and four in Figure 5 and is marked 'Early', while the set for late stage ischaemia (marked 'Late') is found in the final two rows of the figure. The corresponding potentials are in Table 3 (left column), middle and bottom sections, respectively.

Both the Early and Late sets of EPDs are qualitatively the same as the Mean set (Figure 5) in that they progress in the same way from type 1 , through type 2 , to type 3 EPDs as ISC increases. The magnitudes of min1V, maxV and $\min 2 \mathrm{~V}$ are lower, for each ISC value, for the Late set than the Mean set. On the other hand, for the Early set, the magnitudes of min $1 \mathrm{~V}$ and $\operatorname{maxV}$ are slightly larger than their corresponding Mean set values, and the min2V values are comparable with their Early set counterparts.

We repeated this study for two other sets of $f_{p q}$ values. The first had reduced $f_{i q}$ values only $\left(f_{i l}=0.5, f_{i t}=\right.$ $\left.f_{\text {in }}=0.25\right)$ and the second used the values of Barnes [20] $\left(f_{e l}=0.4, f_{i l}=0.9\right)$. In both cases, the EPD pattern for each ISC value was qualitatively the same as in Figure 5, while the potentials lay between those for Early and Late ischaemia in Table 3 (left column).

We also repeated this study using the 'new' representation for the transmembrane potential (equation 5). We found that, although the potentials in each of the Mean, Early and Late sets were reduced relative to Table 3 (Supplementary Table 1), the sets of plots were qualitatively the same (see Supplementary Figure 2) as those in Figure 5 and led to the same conclusions as we have just presented.

\subsection{Mean EPDs for early and late stage ischaemia for the realistic model}

We now repeat the study of Section 3.1 for the realistic model. The corresponding sets of EPDs for the Mean, Early and Late scenarios are given in Figure 7 and the potentials for the EPD features are given in Table 3 (right column). All three sets of EPDs are qualitatively very similar to those in Section 3.1, as they show a progression from type 1 to type 2 and then type 3 EPDs, and the magnitudes of the features increase with ISC. Also, from Table 3, we see that, as for the half-ellipsoidal model, the magnitudes of the Late set are reduced compared with the Mean set, and the magnitudes of $\min 1 \mathrm{~V}$ and $\max \mathrm{V}$ for the Early set are higher than the Mean set, whereas those for $\min 2 \mathrm{~V}$ are not.

The similarities in behaviour, between the half-ellipsoidal model and the realistic model, give us confidence that the half-ellipsoidal model represents realistic behaviour and thus allows us to use the half-ellipsoidal model in later work.

\subsection{Sensitivity of outputs in the half-ellipsoidal model to uncertainty in ischaemic region conductivities}

\subsubsection{Sensitivities}

We showed in Figure 4 that changing the value of $g_{i l}$ in the ischaemic region affected not just the strength of the potentials (as shown in Section 3.1), but the character of the EPD (changing it from type 2 to type 1 ). This information, in addition to the fact that there is considerable 


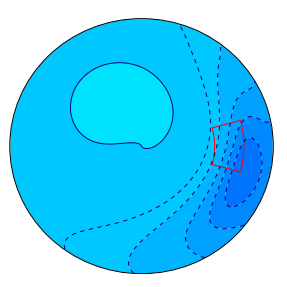

Mean (a) $10 \%$ ischaemia

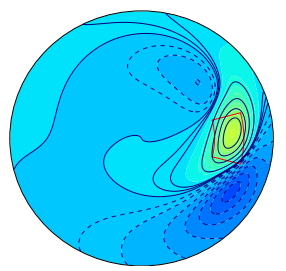

Mean (e) $50 \%$ ischaemia

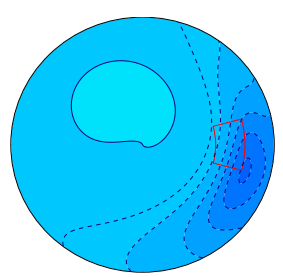

Early (a) $10 \%$ ischaemia

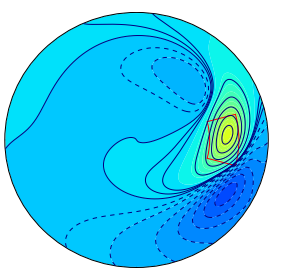

Early (e) $50 \%$ ischaemia

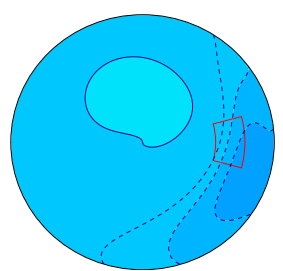

Late (a) $10 \%$ ischaemia

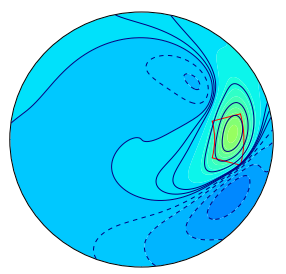

Late (e) $50 \%$ ischaemia

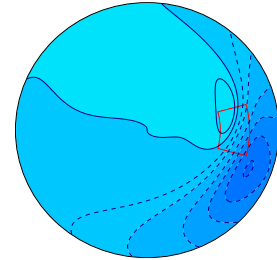

(b) $20 \%$ ischaemia

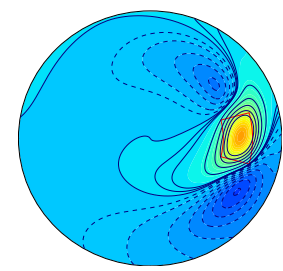

(f) $60 \%$ ischaemia

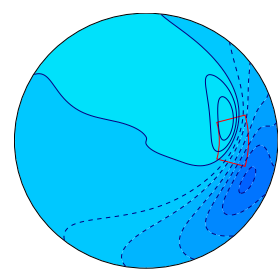

(b) $20 \%$ ischaemia

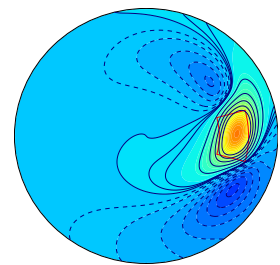

(f) $60 \%$ ischaemia

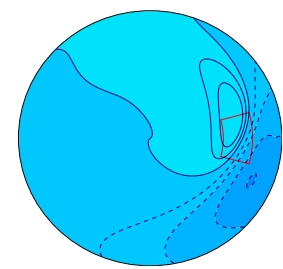

(b) $20 \%$ ischaemia

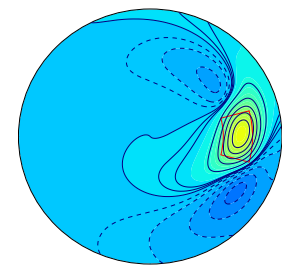

(f) $60 \%$ ischaemia

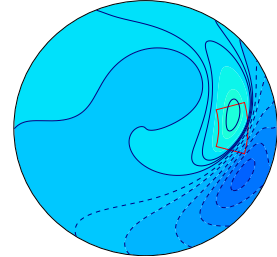

(c) $30 \%$ ischaemia

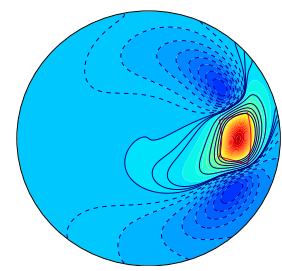

(g) $70 \%$ ischaemia

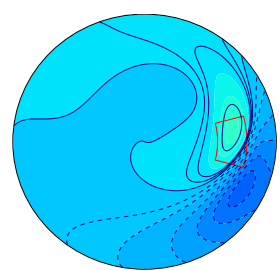

(c) $30 \%$ ischaemia

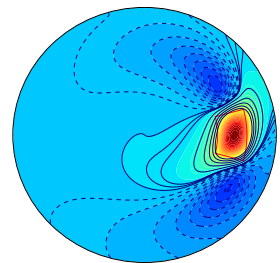

(g) $70 \%$ ischaemia

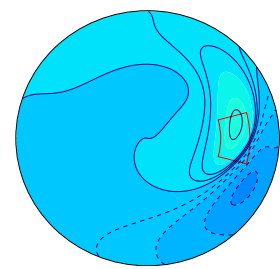

(c) $30 \%$ ischaemia

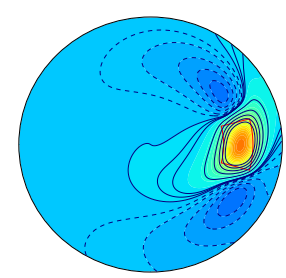

(g) $70 \%$ ischaemia

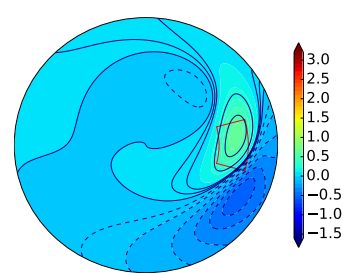

(d) $40 \%$ ischaemia

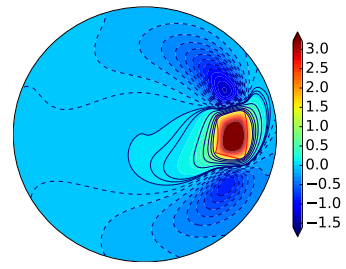

(h) $80 \%$ ischaemia

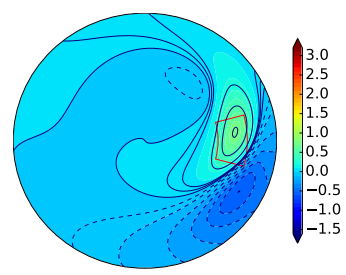

(d) $40 \%$ ischaemia

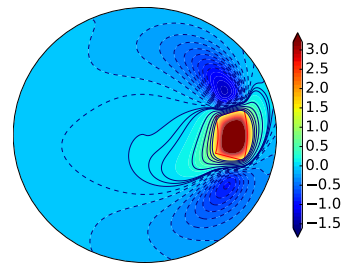

(h) $80 \%$ ischaemia

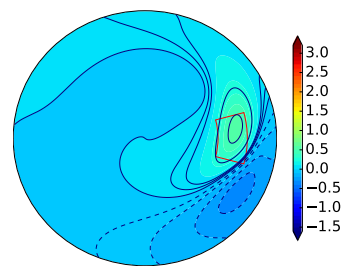

(d) $40 \%$ ischaemia

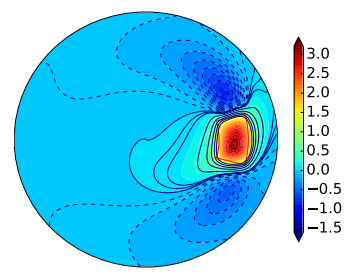

(h) $80 \%$ ischaemia

Figure 5: Epicardial potential distributions generated using mean values for the parameters (Table 2), and also for the early and late stages of ischaemia (Table 1, row 1), for a range of ischaemic depths, for the half-ellipsoidal model. Scale is in mV. See Figure 4 for contour details. 
Table 3: Potentials (in $\mathrm{mV}$ ) for the various features of the epicardial potential distributions for the half-ellipsoidal model, generated using mean values (Table 2) and early and late stages of ischaemia, as in Figure 5.

\begin{tabular}{|c|c|c|c|c|c|c|}
\hline & \multicolumn{3}{|c|}{ Half-ellipsoidal model } & \multicolumn{3}{|c|}{ Realistic model } \\
\hline $\mathrm{ISC}(\%)$ & $\min 1 \mathrm{~V}$ & $\max V$ & $\min 2 \mathrm{~V}$ & $\min 1 \mathrm{~V}$ & $\max V$ & $\min 2 \mathrm{~V}$ \\
\hline \multicolumn{7}{|l|}{ Mean } \\
\hline 10 & -0.49 & - & - & -0.66 & - & - \\
\hline 20 & -0.51 & 0.05 & - & -1.01 & - & - \\
\hline 30 & -0.54 & 0.30 & -0.02 & -0.88 & 0.01 & -0.07 \\
\hline 40 & -0.59 & 0.68 & -0.08 & -0.90 & 0.17 & -0.10 \\
\hline 50 & -0.64 & 1.22 & -0.20 & -0.90 & 0.54 & -0.10 \\
\hline 60 & -0.71 & 2.00 & -0.42 & -0.89 & 1.31 & -0.10 \\
\hline 70 & -0.79 & 2.92 & -0.77 & -1.06 & 2.67 & -0.14 \\
\hline 80 & -0.89 & 4.18 & -1.31 & -1.34 & 4.92 & -0.30 \\
\hline \multicolumn{7}{|c|}{ Early Ischaemia } \\
\hline 10 & -0.51 & - & - & -0.77 & - & - \\
\hline 20 & -0.52 & 0.07 & - & -1.13 & - & - \\
\hline 30 & -0.56 & 0.35 & -0.01 & -0.94 & 0.04 & - \\
\hline 40 & -0.60 & 0.76 & -0.07 & -0.93 & 0.31 & -0.09 \\
\hline 50 & -0.65 & 1.34 & -0.19 & -0.95 & 0.73 & -0.10 \\
\hline 60 & -0.73 & 2.13 & -0.41 & -0.92 & 1.60 & -0.10 \\
\hline 70 & -0.81 & 3.18 & -0.76 & -1.08 & 3.19 & -0.12 \\
\hline 80 & -0.91 & 4.57 & -1.33 & -1.41 & 6.25 & -0.28 \\
\hline \multicolumn{7}{|c|}{ Late Ischaemia } \\
\hline 10 & -0.29 & - & - & -0.37 & - & - \\
\hline 20 & -0.30 & 0.09 & - & -0.58 & - & - \\
\hline 30 & -0.33 & 0.28 & -0.01 & -0.52 & 0.08 & - \\
\hline 40 & -0.36 & 0.57 & -0.03 & -0.52 & 0.29 & -0.05 \\
\hline 50 & -0.40 & 0.95 & -0.11 & -0.50 & 0.63 & -0.05 \\
\hline 60 & -0.44 & 1.48 & -0.24 & -0.51 & 1.23 & -0.04 \\
\hline 70 & -0.49 & 2.17 & -0.46 & -0.63 & 2.44 & -0.05 \\
\hline 80 & -0.55 & 3.11 & -0.83 & -0.95 & 4.19 & -0.09 \\
\hline
\end{tabular}




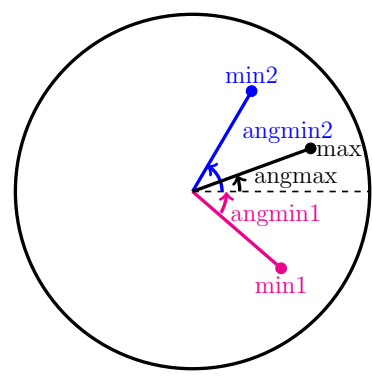

Figure 6: Epicardial potential distribution polar plot features, min1, $\max$, and min2 and their respective angles, angmin1, angmax and angmin2.

uncertainty about the values for the ischaemic region conductivities in both the early and late stages of ischaemia and no real information about ischaemic region normal conductivities, has led us to consider the effect of systematically varying the conductivities in the ischaemic region.

We begin by setting all the model parameters, except for ISC, to their mean values (Table 2), including the conductivities $\left(g_{p q}, p=i, e, q=l, t, n\right)$ outside the ischaemic region, and then allow their corresponding extracellular fractional reduction values $\left(f_{e q}, q=l, t, n\right)$ to vary from 0.1 to 0.9 for the first study (which looks at early stage ischaemia). Then each of the six values $\left(f_{p q}, p=i, e, q=l, t, n\right)$ is varied over the same range for the second study into late stage ischaemia. We use $\mathrm{ISC}=10 \%, 20 \%$ and $60 \%$ to generate EPDs of types 1 , 2 and 3, respectively, and remove EPDs of the wrong type (this was only necessary for the late stage where $\mathrm{ISC}=10 \%$ ). These inputs are summarised in row 2 of Table 1. A LHC sampling routine is used to produce sets of inputs and the model is solved for each set of inputs to produce an EPD, from which we extract the outputs $\min 1 \mathrm{~V}, \max \mathrm{V}$ and $\min 2 \mathrm{~V}$. We then examine the sensitivity (equation (6)) of these outputs to the $f_{p q}$ inputs, using both PLS regression and GP emulators (Section 2.3).

An example of the design data for the first study, where only the $\left(f_{e q}, q=l, t, n\right)$ values are varied, is given in Supplementary Figure 2. This set of plots shows $f_{e l}, f_{e t}$ and $f_{\text {en }}$ plotted against all the outputs that we are considering, bearing in mind that for EPD type 1, for example, $\min 1 \mathrm{~V}$ is the only output. The design data for the second study are also given in the Supplementary material (Figures 3 and 4). In both of the studies, it is not necessary to consider the sensitivity of the angular outputs (angmin1, angmax and angmin2), since in these studies, ROT and ISC are fixed, which has the effect that there is very little variation in the positions of the maximum and minima.

The PLS regression coefficients (Section 2.3) and GP sensitivities (equation (6)) are given in Table 4, where the values in bold are those where the method indicates that an output is particularly sensitive to an input. The results where $\left(f_{e q}, q=l, t, n\right)$ varies, are given in the top half of Table 4 and are labelled 'Early', while the results where $\left(f_{p q}, p=i, e, q=l, t, n\right)$ varies are labelled 'Late' and are given in the bottom half of the Table.
The values for $E^{*} E\{f(x)\}$ are the GP expected means for that particular output. These indicate that for both the Early and Late scenarios the GP mean values for min1V are similar for types 1 and 2 and are less than min $1 \mathrm{~V}$ for type $3, \max V$ (type 2) is low and much less than maxV (type 3 ), and min2 $\mathrm{V}$ is less than min1V (all types). In addition, the Early potentials are all greater than the Late potentials.

Allowing for the fact that the PLS values are signed and the GP values are not, we see that the two methods are consistent in indicating the inputs to which the outputs are most sensitive. In the Early case, these are $f_{e n}$ and $f_{e l}$; that is, every output is particularly sensitive to the conductivities $g_{e n}$ and $g_{e l}$ in the ischaemic region, with the normal conductivities having a stronger effect than the longitudinal conductivities. It can also be seen that there is only one output that is sensitive to $g_{e t}$ in the ischaemic region, and that is $\min 2 \mathrm{~V}$.

The situation is considerably different, however, in the Late case (bottom half of Table 4 ), where $f_{i l}$ is the dominant input. In this case, every output is particularly sensitive to changes in $g_{i l}$ and not at all to the extracellular value $g_{e l}$ that was found in the Early case. In addition, most outputs (all cases but one) have additional sensitivities to either $f_{i n}$ or $f_{e n}$, and, again in the case of min $2 \mathrm{~V}$, to $f_{\text {et }}$.

\subsubsection{Main effect plots}

In addition to calculating GP sensitivity values, we also produced main effect plots $[10,34]$. These plots show the effect, on a particular output, of varying each of the inputs, across the (normalised) range [0,1], while the other inputs are given a mean of 0.5 and a variance of 0.04 . For the Early scenario these plots are given in Figure 8 and for the Late scenario in Figure 9. In each case, the number 0 on the vertical axis represents the emulator expected mean $E^{*} E\{f(x)\}$ for that output, as given in Table 4 . Note that there are different vertical scales on each plot.

These plots provide additional information to the GP sensitivity values, since they indicate the 'direction' of the relationship between the output and the various inputs. For example, in Figure 8, increases in $f_{e l}$ lead to decreases in min1V for all EPD types, and this is consistent with the negative signs of the PLS coefficients in Table 4, for these values. It is worth bearing in mind that, because min $1 \mathrm{~V}$ is negative, this indicates that increases in $f_{e l}$ lead to increases in the magnitude of min $1 \mathrm{~V}$. In fact, examination of the 'direction' of the sensitivities indicates that increases in $f_{e l}$ lead to increases in the magnitude of every output in the Early scenario, although the effect is small for EPD type 2 outputs.

The exact opposite can be seen for $f_{i l}$ in the Late scenario (Figure 9), where increases in $f_{i l}$ lead to a decrease in the magnitude of every output. This is consistent with the positive relationship between $f_{i l}$ and mins 1 and 2 , as well as the negative one for max. In the early case, the increase in the magnitudes of the potentials fits with the 


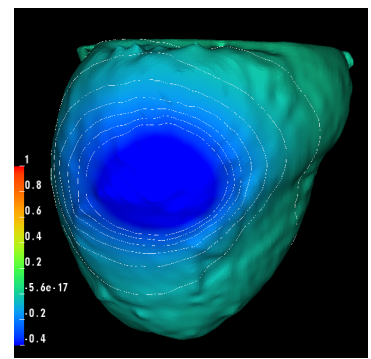

Mean (a) 10\% ischaemia

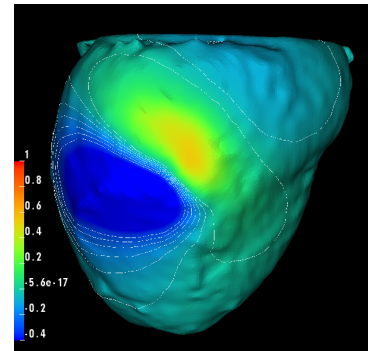

Mean (e) 50\% ischaemia

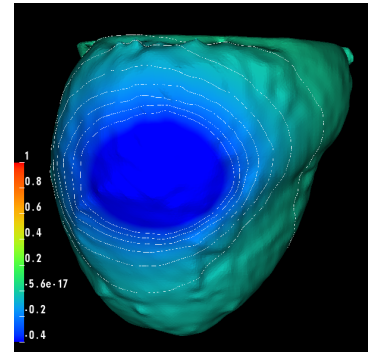

Early (a) $10 \%$ ischaemia

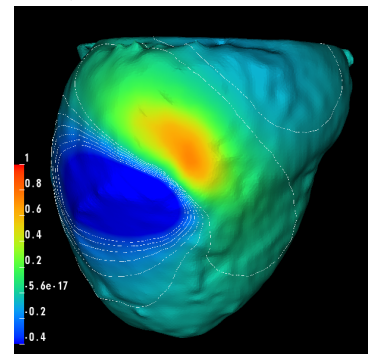

Early (e) $50 \%$ ischaemia

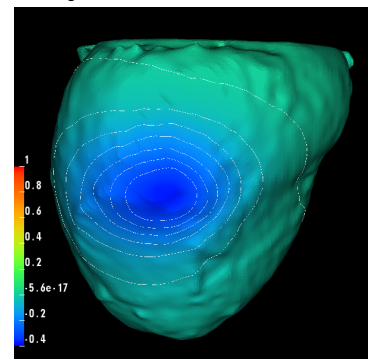

Late (a) $10 \%$ ischaemia

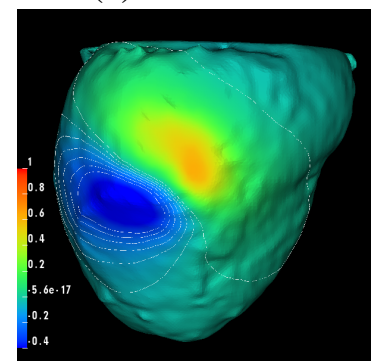

Late (e) $50 \%$ ischaemia

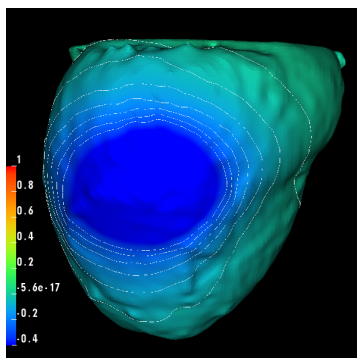

(b) $20 \%$ ischaemia

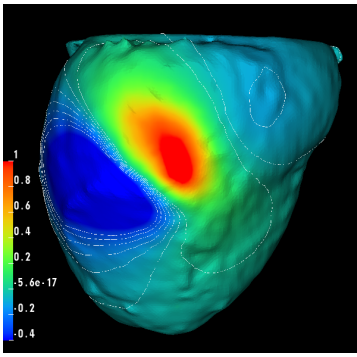

(f) $60 \%$ ischaemia

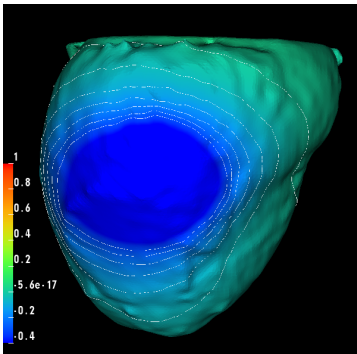

(b) $20 \%$ ischaemia

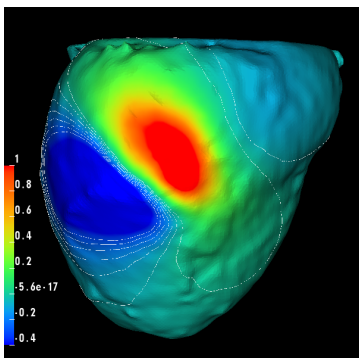

(f) $60 \%$ ischaemia

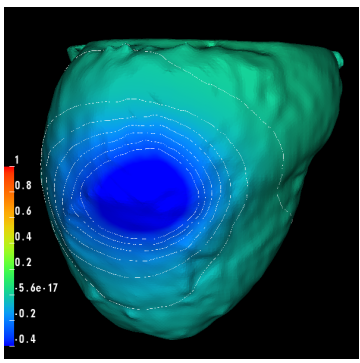

(b) $20 \%$ ischaemia

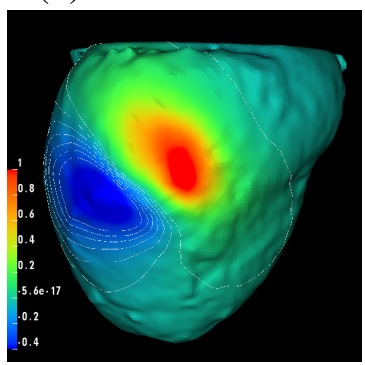

(f) $60 \%$ ischaemia

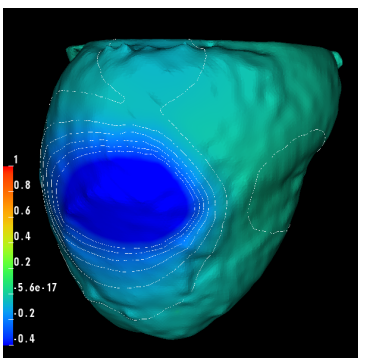

(c) $30 \%$ ischaemia

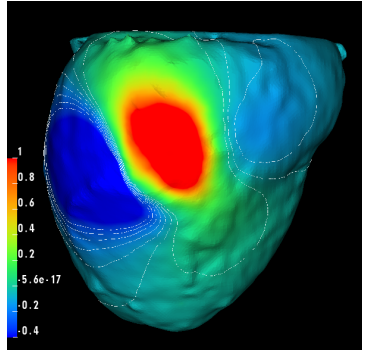

(g) $70 \%$ ischaemia

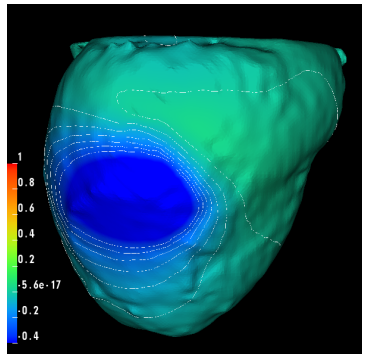

(c) $30 \%$ ischaemia

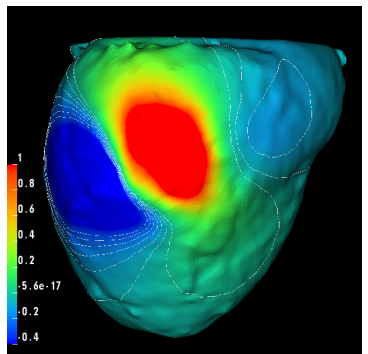

(g) $70 \%$ ischaemia

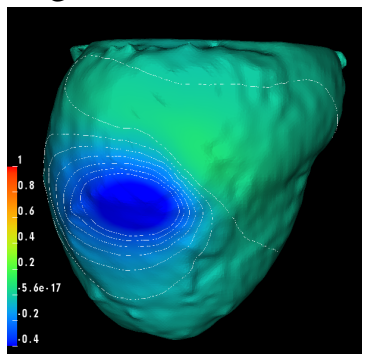

(c) $30 \%$ ischaemia

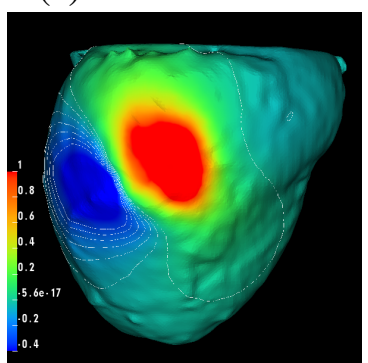

(g) $70 \%$ ischaemia

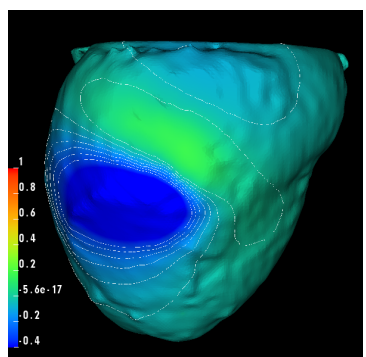

(d) $40 \%$ ischaemia

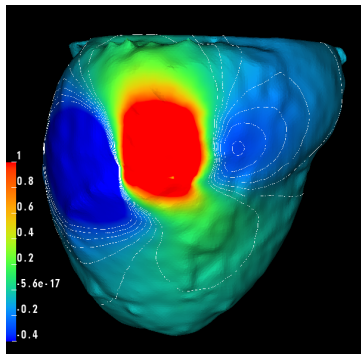

(h) $80 \%$ ischaemia

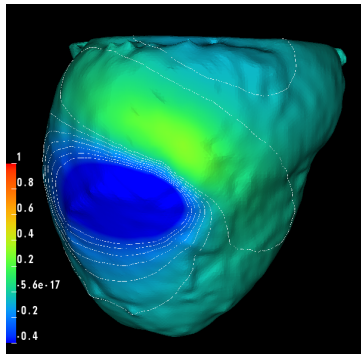

(d) $40 \%$ ischaemia

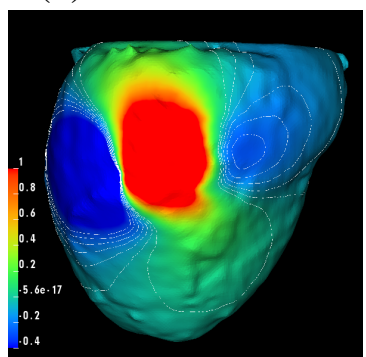

(h) $80 \%$ ischaemia

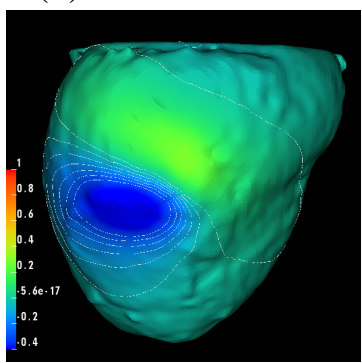

(d) $40 \%$ ischaemia

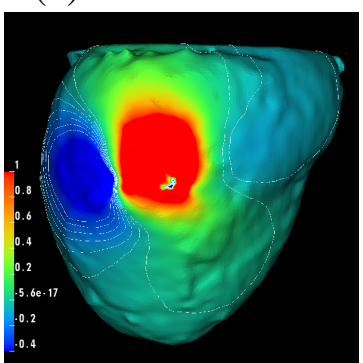

(h) $80 \%$ ischaemia

Figure 7: Epicardial potential distributions generated using mean values for the parameters (Table 2), and also for the early and late stages of ischaemia (Table 1, row 1), for a range of ischaemic depths, for the realistic model. Scale is in mV. Dashed lines are negative potentials, solid lines positive potentials. Contours range from -0.3 to 0 in steps of $0.05 \mathrm{mV}$. 
Table 4: Emulator expected means $E^{*} E\{f(x)\}$ (in $\mathrm{mV}$ ) and sensitivities of the epicardial potential distribution (EPD) feature outputs in the half-ellipsoidal model to reductions in mean conductivities (Table 2) for early (top half of table) and late stage (bottom half) ischaemia. These are calculated as Gaussian Process emulator sensitivities (GP) or by partial least squares (PLS). PLS sensitivities are signed, GP sensitivities are not. Values in bold indicate those inputs to which the output is particularly sensitive.

\begin{tabular}{|c|c|c|c|c|c|c|c|c|c|}
\hline $\begin{array}{l}\text { Early } \\
\text { Features }\end{array}$ & $\begin{array}{l}\text { EPD } \\
\text { Type }\end{array}$ & Method & $E^{*} E\{f(x)\}$ & $f_{i l}$ & $f_{e l}$ & $f_{i t}$ & $f_{\text {et }}$ & $f_{\text {in }}$ & $f_{e n}$ \\
\hline \multirow[t]{2}{*}{$\min 1 \mathrm{~V}$} & \multirow[t]{2}{*}{1} & PLS & & - & -0.58 & - & -0.01 & - & -0.71 \\
\hline & & GP & -0.52 & - & 0.31 & - & 0.00 & - & 0.69 \\
\hline \multirow[t]{2}{*}{$\min 1 \mathrm{~V}$} & \multirow[t]{2}{*}{2} & PLS & & - & -0.53 & - & 0.06 & - & 0.83 \\
\hline & & GP & -0.51 & - & 0.32 & - & 0.00 & - & 0.67 \\
\hline \multirow[t]{2}{*}{$\min 1 \mathrm{~V}$} & \multirow[t]{2}{*}{3} & PLS & & - & -0.43 & - & -0.03 & - & 0.91 \\
\hline & & GP & -0.67 & - & 0.19 & - & 0.00 & - & 0.81 \\
\hline \multirow[t]{2}{*}{$\max V$} & \multirow[t]{2}{*}{2} & PLS & & - & 0.74 & - & 0.18 & - & 0.62 \\
\hline & & GP & 0.08 & - & 0.57 & - & 0.03 & - & 0.39 \\
\hline \multirow[t]{2}{*}{$\max V$} & \multirow[t]{2}{*}{3} & PLS & & - & 0.50 & - & 0.18 & - & -0.81 \\
\hline & & GP & 2.02 & - & 0.28 & - & 0.04 & - & 0.68 \\
\hline \multirow[t]{2}{*}{$\min 2 \mathrm{~V}$} & \multirow[t]{2}{*}{3} & PLS & & - & -0.43 & - & 0.79 & - & 0.67 \\
\hline & & GP & -0.36 & - & 0.15 & - & 0.48 & - & 0.36 \\
\hline Late & EPD & Method & $E^{*} E\{f(x)\}$ & $f_{i l}$ & $f_{e l}$ & $\overline{f_{i t}}$ & $f_{\text {et }}$ & $f_{\text {in }}$ & $f_{\text {en }}$ \\
\hline Features & Type & & & & & & & & \\
\hline \multirow[t]{2}{*}{$\min 1 \mathrm{~V}$} & \multirow[t]{2}{*}{1} & PLS & & 0.81 & -0.07 & 0.04 & -0.01 & 0.36 & 0.01 \\
\hline & & GP & -0.41 & 0.80 & 0.00 & 0.00 & 0.00 & 0.19 & 0.00 \\
\hline \multirow[t]{2}{*}{$\min 1 \mathrm{~V}$} & \multirow[t]{2}{*}{2} & PLS & & 0.91 & -0.10 & 0.03 & 0.00 & 0.26 & 0.30 \\
\hline & & GP & -0.39 & 0.80 & 0.00 & 0.00 & 0.00 & 0.06 & 0.08 \\
\hline \multirow[t]{2}{*}{$\min 1 \mathrm{~V}$} & \multirow[t]{2}{*}{3} & PLS & & 0.81 & -0.22 & 0.01 & -0.03 & 0.07 & 0.58 \\
\hline & & GP & -0.54 & 0.61 & 0.06 & 0.00 & 0.00 & 0.01 & 0.32 \\
\hline \multirow[t]{2}{*}{$\max V$} & \multirow[t]{2}{*}{2} & PLS & & -0.60 & 0.20 & 0.01 & 0.02 & 0.67 & 0.39 \\
\hline & & GP & 0.09 & 0.36 & 0.04 & 0.00 & 0.00 & 0.47 & 0.13 \\
\hline \multirow[t]{2}{*}{$\max V$} & \multirow[t]{2}{*}{3} & PLS & & -0.77 & 0.27 & -0.06 & 0.11 & 0.31 & -0.47 \\
\hline & & GP & 1.75 & 0.59 & 0.09 & 0.00 & 0.01 & 0.09 & 0.21 \\
\hline \multirow[t]{2}{*}{$\min 2 \mathrm{~V}$} & \multirow[t]{2}{*}{3} & PLS & & 0.77 & -0.21 & -0.15 & 0.44 & 0.19 & 0.42 \\
\hline & & GP & -0.29 & 0.54 & 0.05 & 0.02 & 0.17 & 0.04 & 0.18 \\
\hline
\end{tabular}


'direction' of the relationship between $f_{e l}$ and the features (Figure 8) and in a couple of cases ((a) and (d)) for $f_{e n}$, but not in general. This cancelling effect may explain why the increases in the early case are minor.

Another interesting point, which can be seen by comparing Figures 8 and 9, is that the effects of varying the $f_{p q}$ values seem to be independent of one another. For example, if we compare Figure $8(d)$ with Figure $9(d)$, the $f_{\text {eq }}$ values from Figure $8(\mathrm{~d})$ have the same form in Figure $9(\mathrm{~d})$, and this is also true for other pairs like these.

We also repeated this study, but this time using the 'new' transmembrane potential representation (equation (5)) and found that, although in both the Early and Late scenarios the values for the potentials for the various features were reduced, each output was sensitive to exactly the same inputs to those identified in Table 4 (not presented).

In our previous work [10], we found that the effects of ROT and ISC, on the character of the EPD, were much stronger than the effects of the conductivities. So, in the next section, we see if this is also the case here.

\subsection{Sensitivity of outputs in the half-ellipsoidal model to uncertainty in ROT, ISC and ischaemic region con- ductivities}

This study is similar to that in Section 3.3.1, except that we allow ROT and ISC to vary, in addition to the conductivities in the ischaemic region (see row 3 of Table 1 ). As in previous work [10], ROT varies over the range $\left[60^{\circ}, 140^{\circ}\right]$ and two sets of simulations are performed: one with $10^{\circ} \leq$ ISC $\leq 40^{\circ}$ and the other with $40^{\circ} \leq$ ISC $\leq 90^{\circ}$, so that type 1 and 2 EPDs can be extracted from the former and type 3 from the latter.

From the first set of data, for each of the Early and Late cases, we observed that type 1 EPDs could only be found for ISC values up to approximately $20 \%$, rather than up to around $35 \%$ as in our previous work without reduced ischaemic conductivities.

Results for PLS coefficients for this study are given in Table 5, for the Early (top half) and Late (bottom half) scenarios. In this case, we consider two measures for each output - its potential (as in Section 3.3.1), and its position, given as an angle (Figure 6). The fact that there is a variation in the position of the features in this case, but not in Section 3.3.1, comes about because changes in ISC and ROT lead to changes in the position of a feature.

From Table 5, we see that, apart from one case $\left(f_{i l}\right.$ for min1V (type 2)), the only inputs to which the outputs have significant sensitivity are ROT and ISC, indicating that these inputs have a much stronger effect than changes in the ischaemic region conductivities.

Lastly, for completeness, we will also consider the case where the conductivities outside the ischaemic region are also allowed to vary.

\subsection{Sensitivity of outputs in the half-ellipsoidal model to} uncertainty in ROT, ISC and conductivities outside the ischaemic region for early and late ischaemia

In this study, the conductivities outside the ischaemic region, indicated by $g_{p q}$, are allowed to vary across the range (mean $\pm 50 \%$ ) in Table 2 , while the variation of the conductivities in the ischaemic region, as well as that of ROT and ISC, occurs according to row 4 of Table 1.

The results of this work are shown in Table 6 , which summarises the PLS coefficients that were produced. An upward pointing arrow indicates that an increase in the input results in an increase in the output and vice versa for a downward pointing arrow. Only significant relationships are reported (like the bold values in Tables 4 and 5).

We found, once again, that all outputs are sensitive to ROT or ISC or both, with a few outputs sensitive to $g_{i l}, g_{e l}$ or $g_{\text {en }}$. These results are correct for both Early and Late ischaemia, and also for the 'narrow' and the 'new' (Section 2.1) representations for the transmembrane potential. The results are also the same as were found in the previous study [10], where the conductivities were the same inside and outside of the ischaemic region. In addition, by comparing Tables 6 and Table 4, we see that the results are the same for the two cases, for both ROT and ISC.

\section{Discussion}

This work has investigated the role that input parameters play in determining the type of potential distributions that are generated, by subendocardial ischaemia, on the epicardial surface, during the ST segment of the ECG. A number of recent modelling studies [7, 8, 35, 16, 36, $2,5,6,9,10]$ have investigated this, using various modelling assumptions and values for the model inputs. This study has extended this work in a number of different directions: we modelled both early and late stage ischaemia and considered all six bidomain conductivities; we varied the ischaemic region conductivities independently of those in the remainder of the tissue, and, using UQ, we were able to systematically consider the effect of the model inputs on the EPDs. This allowed us to draw some conclusions about the effects of the ischaemic region conductivities, in particular those in the normal direction, despite a lack of knowledge about their values.

We found that reducing the ischaemic region conductivities, to simulate early or late stage ischaemia, resulted in EPDs that were qualitatively the same as simulations that used mean conductivities throughout the whole ventricle (Figure 5 and Supplementary Figure 1), although the values for the potentials had often changed. This was consistent with previous work by Hopenfeld et al. [7], which found that "the basic topography of epicardial potential patterns did not change as a function of conductivity, but that the magnitudes of epicardial potentials are sensitive to the conductivity values" [8].

We found that this was the case regardless of whether we used a representation for the transmembrane potential 


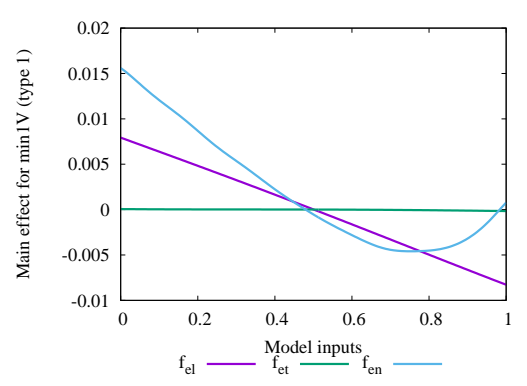

(a) $\min 1 \mathrm{~V}$ (type 1)

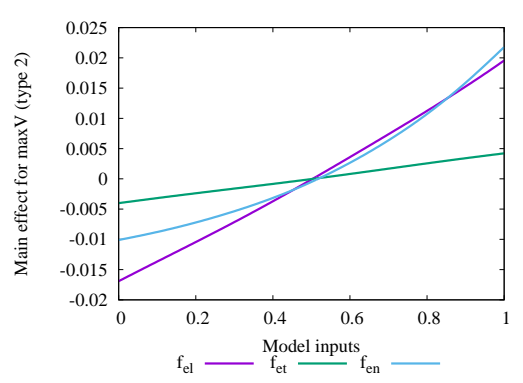

(d) $\max V$ (type 2)

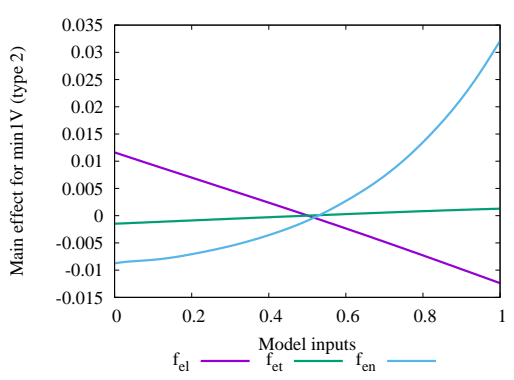

(b) min $1 \mathrm{~V}$ (type 2)

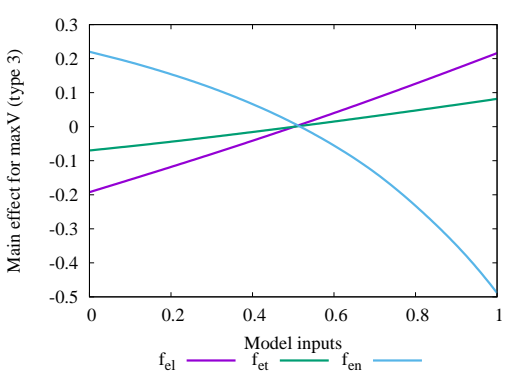

(e) $\max V$ (type 3 )

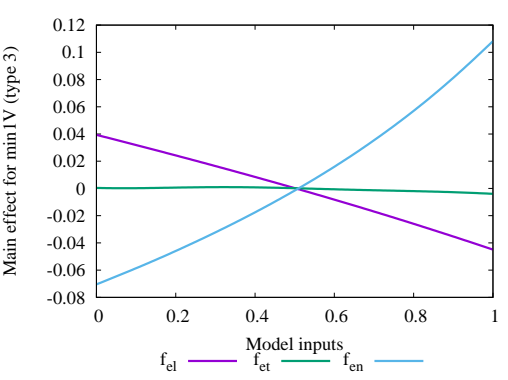

(c) $\min 1 \mathrm{~V}$ (type 3 )

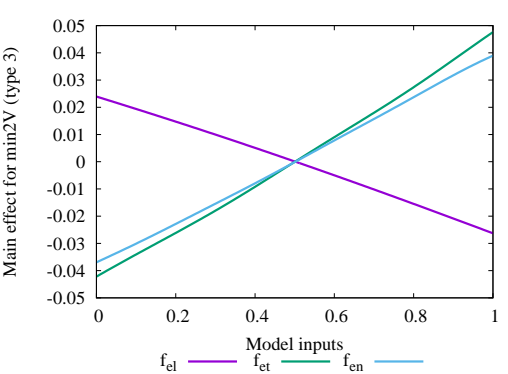

(f) $\min 2 \mathrm{~V}$ (type 3 )

Figure 8: Main effect plots for early ischaemia.

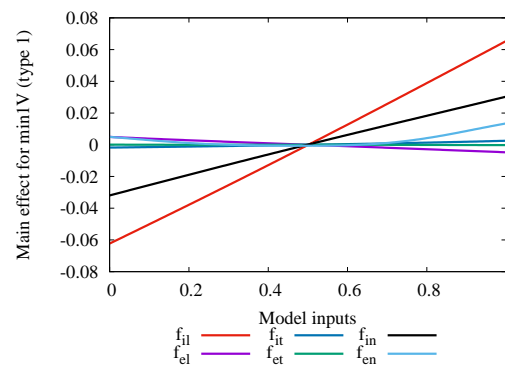

(a) $\min 1 \mathrm{~V}$ (type 1)

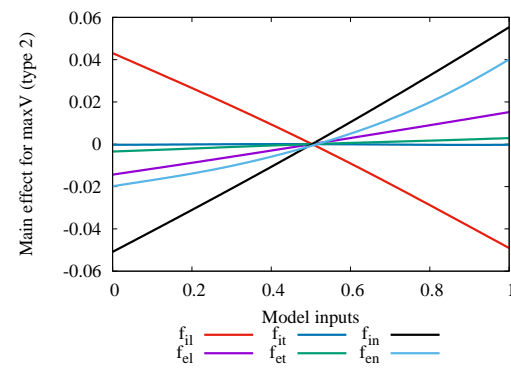

(d) $\max V$ (type 2)

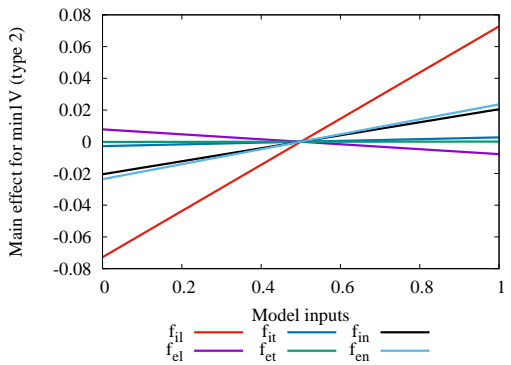

(b) min1V (type 2)

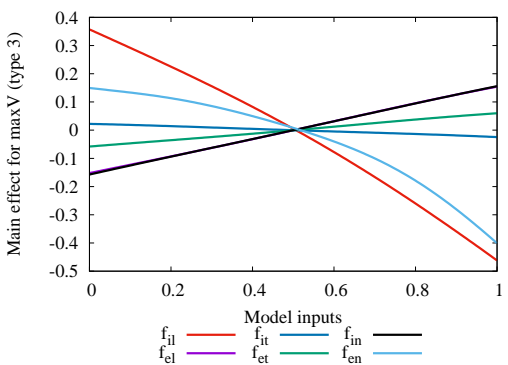

(e) $\max V$ (type 3)

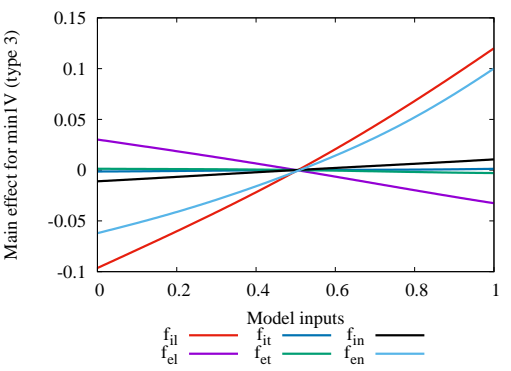

(c) $\min 1 \mathrm{~V}$ (type 3 )

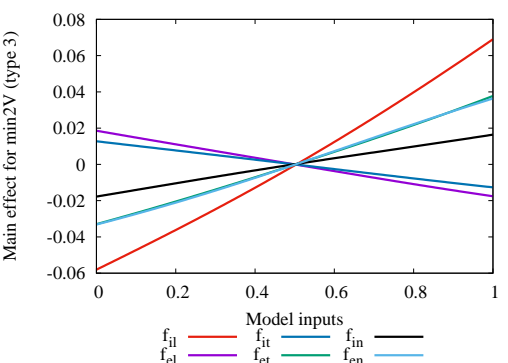

(f) $\min 2 \mathrm{~V}$ (type 3 )

Figure 9: Main effect plots for late ischaemia. 
Table 5: Partial Least Squares sensitivities of the epicardial potential distribution (EPD) features in the half-ellipsoidal model, to fibre rotation (ROT), ischaemic depth (ISC) and six reductions in mean conductivities (Table 2), for early (top half of table) and late stage (bottom half) ischaemia. Values in bold indicate those inputs to which the output is particularly sensitive.

\begin{tabular}{lc|cccccccc}
\hline Features & EPD & ROT & ISC & $f_{i l}$ & $f_{\text {el }}$ & $f_{i t}$ & $f_{\text {et }}$ & $f_{\text {in }}$ & $f_{\text {en }}$ \\
Early & Type & & & & & & & & \\
\hline $\min 1 \mathrm{~V}$ & 1 & $\mathbf{- 0 . 9 7}$ & 0.05 & - & -0.01 & - & 0.01 & - & 0.04 \\
& 2 & $\mathbf{- 0 . 8 9}$ & -0.21 & - & -0.08 & - & 0.01 & - & -0.01 \\
& 3 & $\mathbf{- 0 . 7 7}$ & $\mathbf{- 0 . 4 7}$ & - & -0.13 & - & 0.02 & - & 0.32 \\
maxV & 2 & 0.01 & $\mathbf{0 . 9 7}$ & - & 0.10 & - & 0.02 & - & 0.04 \\
& 3 & 0.06 & $\mathbf{0 . 9 6}$ & - & 0.11 & - & 0.06 & - & 0.20 \\
$\min 2 \mathrm{~V}$ & 3 & 0.31 & $\mathbf{- 0 . 9 2}$ & - & -0.03 & - & 0.08 & - & 0.06 \\
\hline $\min 1 \mathrm{~A}$ & 1 & $\mathbf{0 . 7 7}$ & $\mathbf{- 0 . 4 1}$ & - & -0.05 & - & -0.02 & - & 0.00 \\
& 2 & $\mathbf{1 . 0 2}$ & $\mathbf{- 0 . 6 4}$ & - & -0.02 & - & 0.01 & - & -0.01 \\
& 3 & $\mathbf{0 . 9 3}$ & $\mathbf{- 0 . 1 6}$ & - & -0.03 & - & 0.06 & - & -0.06 \\
$\operatorname{maxA}$ & 2 & 0.24 & $\mathbf{- 0 . 7 4}$ & - & 0.02 & - & 0.01 & - & 0.01 \\
& 3 & 0.34 & $\mathbf{- 0 . 9 1}$ & - & 0.03 & - & 0.03 & - & -0.02 \\
$\min 2 \mathrm{~A}$ & 3 & 0.18 & $\mathbf{- 0 . 9 8}$ & - & 0.01 & - & 0.09 & - & -0.03 \\
\hline Late & & & & & & & & & \\
\hline $\min 1 \mathrm{~V}$ & 1 & $\mathbf{- 0 . 8 9}$ & 0.03 & 0.18 & 0.00 & 0.06 & 0.00 & 0.16 & 0.05 \\
& 2 & $\mathbf{- 0 . 7 6}$ & -0.21 & $\mathbf{0 . 4 7}$ & -0.06 & 0.00 & 0.00 & 0.08 & 0.12 \\
& 3 & $\mathbf{- 0 . 6 6}$ & -0.41 & 0.39 & -0.14 & 0.00 & 0.02 & 0.06 & 0.29 \\
$\max \mathrm{V}$ & 2 & -0.01 & $\mathbf{0 . 9 6}$ & -0.21 & 0.07 & 0.01 & 0.02 & 0.20 & 0.09 \\
& 3 & 0.05 & $\mathbf{0 . 8 8}$ & -0.19 & 0.07 & -0.02 & 0.03 & 0.05 & -0.17 \\
$\min 2 \mathrm{~V}$ & 3 & 0.31 & $\mathbf{- 0 . 8 5}$ & 0.12 & -0.06 & -0.02 & 0.00 & 0.02 & 0.10 \\
\hline $\min 1 \mathrm{~A}$ & 1 & $\mathbf{0 . 6 9}$ & $\mathbf{- 0 . 5 1}$ & 0.26 & -0.07 & -0.04 & -0.03 & -0.29 & -0.03 \\
& 2 & $\mathbf{1 . 0 6}$ & $\mathbf{- 0 . 6 9}$ & 0.02 & -0.02 & -0.04 & 0.01 & -0.05 & -0.03 \\
$\max$ & 3 & $\mathbf{0 . 9 3}$ & $\mathbf{- 0 . 1 4}$ & -0.03 & 0.01 & -0.02 & 0.03 & -0.05 & -0.10 \\
$\min 2 \mathrm{~A}$ & 2 & 0.09 & $\mathbf{- 0 . 6 5}$ & 0.01 & 0.00 & 0.03 & 0.01 & -0.18 & -0.09 \\
& 3 & 0.36 & $\mathbf{- 0 . 9 2}$ & 0.00 & 0.01 & 0.02 & 0.00 & -0.04 & 0.00 \\
& 3 & 0.17 & $\mathbf{- 0 . 9 7}$ & 0.01 & 0.03 & -0.04 & 0.07 & 0.07 & -0.02 \\
& & & & & & & & &
\end{tabular}

Table 6: Summary of the epicardial potential distribution (EPD) feature outputs (rows) and the input variables (columns) to which they are sensitive, for the half-ellipsoidal model. ROT=fibre rotation and ISC=ischaemic depth. The indicated inputs are significant for both the early and late stages of ischaemia and for both the 'narrow' and 'new' representations of the transmembrane potential. Blank spaces indicate no significant relationship. An increase in the input variable that results in an increase in the output is represented by an upward pointing arrow and an increase in the input that results in a decrease in the output is represented by a downward pointing arrow.

\begin{tabular}{lc|ccccccccc}
\hline Feature & EPD & ROT & ISC & $g_{b}$ & $g_{i l}$ & $g_{\text {el }}$ & $g_{i t}$ & $g_{\text {et }}$ & $g_{\text {in }}$ & $g_{\text {en }}$ \\
& Type & & & & & & & & & \\
\hline $\min 1 \mathrm{~V}$ & 1 & $\downarrow$ & & & & & & & & \\
& 2 & $\downarrow$ & & & $\downarrow$ & & & & & \\
& 3 & $\downarrow$ & & & & $\uparrow$ & & & & \\
$\operatorname{maxV}$ & 2 & & $\uparrow$ & & & & & & & $\uparrow$ \\
& 3 & & $\uparrow$ & & & & & & & \\
$\min 2 \mathrm{~V}$ & 3 & & $\downarrow$ & & & & & & \\
\hline angmin 1 & 1 & $\uparrow$ & $\downarrow$ & & & & & & & \\
& 2 & $\uparrow$ & $\downarrow$ & & & & & & & \\
& 3 & $\uparrow$ & & & & & & & & \\
$\operatorname{angmax}$ & 2 & & $\downarrow$ & & & & & & & $\downarrow$ \\
& 3 & & $\downarrow$ & & & & & & & \\
$\operatorname{angmin} 2$ & 3 & & $\downarrow$ & & & & & & & \\
\hline
\end{tabular}


that resulted in a 'narrow' border region or used a 'new' representation (Figure 3). In either of the late cases or the early case with the 'new' representation (Table 3 and Supplementary Table 1), the magnitudes of the potentials were reduced compared with the mean case and the late case potentials were reduced compared with the early case (fourth column of Table 4). These late case results are consistent with experimental work [18]. This result may be important to note, as smaller potentials may be harder to detect on the epicardial surface. However, we also found that for the early case (with the 'narrow' border) the magnitudes of the potentials for min1 and max were greater by a small amount than the mean case. Together these two results may explain the findings from the experimental work of Aras et al. [37], who found that, in some cases, ischaemia could not be detected on the epicardial surface.

We also found that type 1 EPDs (a single minimum) were unlikely to occur for early or late ischaemia for ischaemic depths of greater than 20\% (unlike previous work by ourselves [10] and Potse et al. [2], where the value was around 30-40\%), which may also be important in terms of detection.

As in our previous study [10] that used the half-ellipsoidal model with the same conductivities throughout the ventricle, we again found that ROT and ISC were the most significant model inputs, in terms of the magnitudes and positions of the EPD features that were produced. Although these results do not have a direct clinical application, they do provide insight into parameters used in models that seek to understand the connection between ischaemia and epicardial potentials, which is a necessary first step before moving to torso potentials and the ECG.

For example, since ST depression in various leads is used diagnostically for ischaemia [1] and prognostically for the effects of ischaemia [38], our finding about the relationship between ROT and min1 (both magnitude and position) has important modelling consequences, especially as this result holds regardless of assumptions about transmembrane potential representation or choice of conductivity values. The consequence of the connection between min1 and ROT is that it seems to be essential that more sophisticated models should consider a range of fibre rotation values, in order to represent the variation in the population [39]. This is in addition to considering a range ischaemic depths, since we found that both max and min2 are sensitive to that parameter (again regardless of the above assumptions).

This is not to say that conductivity values are unimportant, given, for example, that we have shown that the magnitude of all of the EPD features (min1, max, min2) is lower, when the conductivities are reduced to simulate late stage ischaemia, than when simulating early stage ischaemia (Table 4). Also studies have shown the importance of conductivity values when modelling other heart conditions, such as heart failure or hypertrophy. For example, a recent UQ study by Sanchez et al. [40] found that some of the bidomain conductivities are important param- eters when tuning a realistic anatomical-electrophysiological model of the heart. It may just be that conductivity values are more important at the stage of "fitting" subjectspecific models, rather than for more general studies that seek to represent the range of possible EPDs.

There are a number of limitations to this study, including the use of only the half-ellipsoidal model for the sensitivity study (although this is partially justified by the similarities in the form of the EPD between that model and the more realistic model, at least when mean values are used and early and late ischaemia are simulated). Although in this work we did not study the effect of varying the size and position of the ischaemic patch, we did do this in our previous work with the same model $[3,10]$ where the conductivities did not differ between the ischaemic patch and the rest of the tissue, and, in that case, we found that the EPDs were qualitatively the same and the outputs were found to be sensitive to exactly the same inputs as in the original model.

We also did not consider the effect of varying the shape of the ischaemic region; however, previous work [20], with the same model and Clerc's [41] conductivities used throughout the ventricle, found that using a cylindrical patch results in similar EPDs, whereas an ellipsoidal patch results in the second minimum developing later. This could be an avenue for future work. Another avenue for future work could be a study of partial thickness, rather than subendocardial ischaemia. This approach is suggested by the recent experimental work of Aras et al. [37], which found that ischaemia appears to originate throughout the ventricular wall rather than solely at the endocardium. Other future work could include coupling a realistic heart model with a torso model to consider potentials on the body surface, rather than the epicardial surface.

\section{Conclusion}

In this work, we modelled both early and late stage subendocardial ischaemia during the ST segment by varying the conductivities in the ischaemic region compared with the remainder of the ventricle. We used a half-ellipsoidal model and a more realistic model and found that the EPD pattern changed consistently from a minimum over the ischaemic region, to a maximum there flanked by a minimum, followed by these two plus another minimum. This was exactly the same progression as in previous work where the conductivities were taken to be the same inside and outside the ischaemic region. The difference was that the potentials were lower in magnitude for late stage ischaemia, which may make them more difficult to detect.

Using uncertainty quantification techniques, we found that the effects of conductivity changes were minor in comparison to the effects of fibre rotation and ischaemic depth. Since we also found that the position and magnitude of the first minimum to appear is very sensitive to fibre rotation, we suggest that it is essential to include a range of fibre rotation values in modelling studies to allow for 
variation in the population. This would allow such studies to investigate whether this range is part of the reason for variations in the presentation of depressions on the heart surface in experimental studies, and perhaps also on the torso, leading to effects on the ECG.

\section{References}

[1] K. Akkerhuis, M. Simoons, Comprehensive electrocardiology, no. 36, Springer-Verlag, London, 2011, Ch. Exercise electrocardiography and exercise testing, pp. 1677-1719.

[2] M. Potse, R. Coronel, S. Falcao, A. R. LeBlanc, A. Vinet, The effect of lesion size and tissue remodeling on ST deviation in partial-thickness ischemia;, Heart Rhythm 4 (2) (2007) 200-206.

[3] B. M. Johnston, P. R. Johnston, Using generalised polynomial chaos to examine various parameters in a half-ellipsoidal ventricular model of partial thickness ischaemia, in: C. Pickett (Ed.), Computing in Cardiology, Vol. 44, 2017, pp. 268-024.

[4] J. P. Barnes, P. R. Johnston, The effect of ischaemic region shape on ST potentials using a half-ellipsoid model of the left ventricle, in: A. Murray (Ed.), Computing in Cardiology, Vol. 39, IEEE, IEEE Press, 2012, pp. 461-464.

[5] J. P. Barnes, P. R. Johnston, How ischaemic region shape affects ST potentials in models of cardiac ischaemia, Mathematical Biosciences 239 (213-221).

[6] B. M. Burton, J. D. Tate, W. Good, R. S. MacLeod, The role of reduced left ventricular, systolic blood volumes in ST segment potentials overlying diseased tissue of the ischaemic heart, in: A. Murray (Ed.), Computing in Cardiology, Vol. 43, 2016, pp. 209-212.

[7] B. Hopenfeld, J. G. Stinstra, R. S. MacLeod, Mechanism for ST depression associated with contiguous subendocardial ischaemia, Journal of Cardiovascular Electrophysiology 15 (2004) $1200-1206$.

[8] B. Hopenfeld, J. G. Stinstra, R. S. MacLeod, The effect of conductivity on ST-segment epicardial potentials arising from subendocardial ischemia, Annals of Biomedical Engineering 33 (6) (2005) 751-763.

[9] B. M. Johnston, S. Coveney, E. T. Y. Chang, P. R. Johnston, R. H. Clayton, Quantifying the effect of uncertainty in input parameters in a simplified bidomain model of partial thickness ischaemia, Medical and Biological Engineering and Computing 56 (2018) 761-780.

[10] B. M. Johnston, P. R. Johnston, Determining the most significant input parameters in models of subendocardial ischaemia and their effect on st segment epicardial potential distributions, Computers in Biology and Medicine 95 (2018) 75-89.

[11] L. Tung, A bi-domain model for describing ischaemic myocardial D-C potentials, Ph.D. thesis, Massachusetts Institute of Technology (June 1978).

[12] F. B. Sachse, A. Moreno, G. Seemann, J. Abildskov, A model of electrical conduction in cardiac tissue including fibroblasts, Annals of Biomedical Engineering 37 (2009) 874-889.

[13] A. Quaglino, S. Pezzuto, P.-S. Koutsourelakis, A. Auricchio, R. Krause, Fast uncertainty quantification of activation sequences in patient-specific cardiac electrophysiology meetign clinical time constraints, International Journal for Numerical Methods in Biomedical Engineering (2018) DOI: $10.1002 / \mathrm{cnm} .2985$.

[14] J. Greiner, A. C. Sankarankutty, G. Seemann, T. Seidel, F. B. Sachse, Confocal microscopy-based estimaton of parameters for computational modeling of electrical conduction in the normal and infarcted heart, Frontiers in Physiology 9 (239) (2018) 1-15.

[15] P. R. Johnston, Cardiac conductivity values - a challenge for experimentalists?, Noninvasive Functional Source Imaging of the Brain and Heart \& 2011 8th International Conference on Bioelectromagnetism (NFSI \& ICBEM) (2011) 39-43.

[16] J. G. Stinstra, S. Shome, B. Hopenfeld, R. S. MacLeod, Modelling passive cardiac conductivity during ischaemia, Medical \& Biological Engineering \& Computing 43 (6) (2005) 776-782.
[17] A. G. Kleber, C. B. Riegger, Electrical constants of arterially perfused rabbit papillary muscle, Journal of Physiology 385 (1987) 307-324.

[18] W. Smith, W. Fleet, F. Johnson, T. Engle, C. Cascio, The ib phase of ventricular arrhythmias in ischemic in situ porcine heart is related to changes in cell-to-cell electrical coupling, Circulation 92 (1995) 3051-3060.

[19] E. Boccia, S. Luther, U. Parlitz, Modelling far field pacing for terminating spiral waves pinned to ischaemic heterogeneities in cardiac tissue, Philosophical Transactions of the Royal Society A 375 (2096) (2017) 20160289.

[20] J. P. Barnes, Mathematically modeling the electrophysiological effects of ischaemia in the heart, Ph.D. thesis, Griffith University, Brisbane, Australia (2013).

[21] W. Becker, J. E. Oakley, C. Surace, P. Gili, J. Rowson, K. Worden, Bayesian sensitvity analysis of a nonlinear finite element model, Mechanical Systems and Signal Processing 32 (2012) $18-31$.

[22] J. E. Oakley, A. O'Hagan, Probabilistic sensitivity analysis of complex models: a Bayesian approach, Journal of the Royal Statistical Society: Series B (Statistical Methodology) 66 (751$769)$.

[23] H. Abdi, Partial least squares regression (PLS-regression), Encyclopedia for research methods for the social sciences, Sage, 2003, pp. 792-795.

[24] E. Sobie, Parameter sensitivity analysis in electrophysiological models using multivariate regression, Biophysical Journal 96 (2009) 1264-1274.

[25] D. Xiu, J. S. Hesthaven, High-order collocation methods for differential equations with random inputs, SIAM Journal on Scientific Computing 27 (3) (2005) 1118-1139.

[26] D. Xiu, Efficient collocational approach for parametric uncertainty analysis, Communications in Computational Physics 2 (2) (2007) 293-309.

[27] P. R. Johnston, A finite volume method solution for the bidomain equations and their application to modelling cardiac ischaemia, Computer Methods in Biomechanics and Biomedical Engineering 13 (2) (2010) 157-170.

[28] P. Colli-Franzone, L. Guerri, Spreading of excitation in 3-D models of the anisotropic cardiac tissue I: Validation of the eikonal model, Mathematical Biosciences 113 (1993) 145-209.

[29] SCIRun: A Scientific Computing Problem Solving Environment, Scientific Computing and Imaging Institute (SCI). [link]. URL http://www. scirun.org

[30] P. R. Johnston, A cylindrical model for studying subendocardial ischaemia in the left ventricle, Mathematical Biosciences 186 (1) (2003) 43-61.

[31] R. Coronel, F. J. G. Wilms-Schopman, T. Opthof, F. J. L. van Capelle, M. J. Janse, Injury current and gradients of diastolic stimulation threshold, TQ potential, and extracellular potassium concentration during acute regional ischemia in the isolated perfused pig heart, Circulation Research 68 (1991) 12411249.

[32] M. Potse, R. Coronel, A. R. LeBlanc, A. Vinet, The role of extracellular potassium transport in computer models of the ischemic zone, Medical and Biological Engineering and Computing 45 (12) (2007) 1187-1199.

[33] P. R. Johnston, D. Kilpatrick, C. Y. Li, The importance of anisotropy in modelling ST segment shift in subendocardial ischaemia, IEEE Transactions on Biomedical Engineering 48 (12) (2001) 1366-1376.

[34] E. T. Y. Chang, M. Strong, R. H. Clayton, Bayesian sensitivity analysis of a cardiac cell model using a Gaussian Process emulator, PLoS ONE 10 (6) (2015) e0130252.

[35] M. C. MacLachlan, J. Sundnes, G. T. Lines, Simulation of ST segment changes during subendocardial ischemia using a realistic 3-D cardiac geometry, IEEE Transactions on Biomedical Engineering 52 (5) (2005) 799-807.

[36] M. Potse, A.-R. LeBlanc, R. Cardinal, A. Vinet, ST elevation or depression in subendocardial ischemia?, in: 28th IEEE EMBS Annual International Conference, 2006, pp. 3899-3902. 
[37] K. Aras, B. Burton, D. Swenson, R. MacLeod, Spatial organisation of acute myocardial ischaemia, Journal of Electrocardiology 49 (2016) 323-336

[38] M. I. Hayiroğlu, M. Keskin, A. O. Uzun, C. Türkkan, A. I. Tekkeşin, O. Kozan, What is the predictive value of ST segment depression in inferior leads in first acute anterior myocardial infarction, Journal of Electrocardiology 51 (2018) 524-530.

[39] H. Lombaert, J. Peyrat, P. Croisille, S. Rapacchi, L. Fanton, F. Cheriet, P. Clarysse, I. Magnin, H. Delingette, N. Ayache, Human atlas of the cardiac fiber architecture: study on a healthy population, IEEE Transactions on Medical Imaging 31 (7) (2012) 1436-1447.

[40] C. Sanchez, G. D'Ambrosio, F. Maffessanti, E. G. Caiani, F. W. Prinzen, R. Krause, A. A, M. Potse, Sensitivity analysis of ventricular activation and eletrocardiogram in tailored models of heart-failure patients, Medical and Biological Engineering and Computing 56 (2018) 491-504.

[41] L. Clerc, Directional differences of impulse spread in trabecular muscle from mammalian heart, Journal of Physiology 255 (1976) 335-346. 